\title{
SPECTRAL BEHAVIOUR OF QUASIANALYTIC CONTRACTIONS
}

\author{
LÁSZLÓ KÉRCHY AND ATTILA SZALAI \\ (Communicated by Pamela Gorkin)
}

\begin{abstract}
We pose, and answer partially, questions in connection with the spectral behaviour of quasianalytic contractions. These problems are related to the hyperinvariant subspace problem in the class of asymptotically nonvanishing contractions.
\end{abstract}

Let $\mathcal{H}$ be a separable, complex Hilbert space, and let $\mathcal{L}(\mathcal{H})$ denote the $C^{*}$ algebra of bounded, linear operators acting on $\mathcal{H}$. For any $T \in \mathcal{L}(\mathcal{H})$, Lat $T$ means the lattice of all invariant subspaces of $T$, while Hlat $T$ stands for the lattice of all hyperinvariant subspaces of $T$. We recall that a subspace (i.e. closed linear manifold) $\mathcal{M}$ is hyperinvariant for $T$, if it is invariant for every operator $C$ in the commutant $\{T\}^{\prime}=\{A \in \mathcal{L}(\mathcal{H}): A T=T A\}$ of $T$, i.e. $C \mathcal{M} \subset \mathcal{M}$. The Invariant Subspace Problem (ISP) asks whether Lat $T$ is non-trivial, i.e. Lat $T \neq\{\{0\}, \mathcal{H}\}$, for every $T \in \mathcal{L}(\mathcal{H})$. The Hyperinvariant Subspace Problem (HSP) asks whether Hlat $T$ is non-trivial, provided $T$ is not a scalar multiple of the identity operator $I$. These problems are arguably the most challenging open questions in operator theory. They can be reduced to the case when $T$ is an absolutely continuous (a.c.) contraction, that is $\|T\| \leq 1$ and $T$ splits into the orthogonal sum $T=U \oplus T_{c}$, where $U$ is a unitary operator whose spectral measure is a.c. with respect to the normalized Lebesgue measure $m$ on the unit circle $\mathbb{T}$, and $T_{c}$ is completely nonunitary, i.e. the restriction of $T_{c}$ to any non-zero invariant subspace is not unitary.

We focus on the particular case when the a.c. contraction $T$ is asymptotically non-vanishing (a.n.v.), that is when $\lim _{n \rightarrow \infty}\left\|T^{n} h\right\|>0$ holds for some vector $h \in \mathcal{H}$. Then a non-trivial unitary asymptote can be associated with $T$, which is a pair $(X, V)$, where $V$ is an a.c. unitary operator acting on a non-zero Hilbert space $\mathcal{K}, X$ is a bounded linear transformation from $\mathcal{H}$ into $\mathcal{K},\|X h\|=\lim _{n \rightarrow \infty}\left\|T^{n} h\right\|$ holds for all $h \in \mathcal{H}, X T=X V$ and $\vee_{n=1}^{\infty} V^{-n} X \mathcal{H}=\mathcal{K}$. The pair $(X, V)$ is unique up to isomorphism; for details see Chapter IX in [NFBK], and [Ker13.

Received by the editors February 04, 2014.

2010 Mathematics Subject Classification. Primary 47A10, 47A15, 47A60.

Key words and phrases. Quasianalytic contraction, spectrum, hyperinvariant subspace.

The first author was supported by the European Union and co-funded by the European Social Fund under the projects "Telemedicine-focused research activities on the field of Mathematics, Informatics and Medical Sciences" of project number TÁMOP-4.2.2.A-11/1/KONV-2012-0073, and "Impulse lasers for use in materials science and biophotonics" of project number TÁMOP4.2.2.A-11/1/KONV-2012-0060.

The second named author was supported by the European Union and the State of Hungary, co-financed by the European Social Fund in the framework of TÁMOP 4.2.4. A/2-11-1-2012-0001 'National Excellence Program'. 
The residual set $\omega(T)$ of $T$ is the measurable support of the spectral measure $E$ of $V$, which means that $E(\alpha)=0$ if and only if $m(\alpha \cap \omega(T))=0$. Clearly, $m(\omega(T))>0$.

Another important spectral invariant is connected with the Sz.-Nagy-Foias functional calculus $\Phi_{T}: H^{\infty} \rightarrow \mathcal{L}(\mathcal{H})$ for $T$. For the definition and basic properties of $\Phi_{T}$ see Chapter III in [NFBK]. We note that $\Phi_{T}$ is monotone, that is $f_{2} \stackrel{a}{\prec} f_{1}$ (i.e. $\left|f_{2}(z)\right| \leq\left|f_{1}(z)\right|$ for all $z$ in the open unit disc $\mathbb{D}$ ) implies $f_{2}(T) \stackrel{a}{\prec} f_{1}(T)$ (i.e. $\left\|f_{2}(T) h\right\| \leq\left\|f_{1}(T) h\right\|$ for all $\left.h \in \mathcal{H}\right)$. Taking a decreasing sequence $F=\left\{f_{n}\right\}_{n=1}^{\infty}$ in $H^{\infty}$, let us consider the limit function $\varphi_{F}(\zeta)=\lim _{n \rightarrow \infty}\left|f_{n}(\zeta)\right|$ defined for a.e. $\zeta \in \mathbb{T}$, the measurable set $N_{F}=\left\{\zeta \in \mathbb{T}: \varphi_{F}(\zeta)>0\right\}$, and the hyperinvariant subspace $\mathcal{H}_{0}(T, F)=\left\{h \in \mathcal{H}: \lim _{n \rightarrow \infty}\left\|f_{n}(T) h\right\|=0\right\}$. The quasianalytic spectral set $\pi(T)$ of $T$ is the largest measurable set on $\mathbb{T}$ with the property that $\mathcal{H}_{0}(T, F)=\{0\}$ whenever $m\left(N_{F} \cap \pi(T)\right)>0$. The a.n.v. contraction $T$ is called quasianalytic, if $\pi(T)=\omega(T)$. (Note that $\pi(T) \subset \omega(T)$ always holds.) The class of quasianalytic contractions was introduced and studied in the papers Ker01, Ker11, Ker13, KT12 and KSz. Though (ISP) and (HSP) are open for a.n.v. contractions, the following result of Ker01 shows that these questions are settled in the non-quasianalytic case.

Proposition 1. If $T$ is an a.n.v. contraction which is not quasianalytic, then $T$ has a non-trivial hyperinvariant subspace.

By this proposition, it becomes crucial to determine the spectral behaviour of quasianalytic contractions. Namely, if an a.n.v. contraction $T$ does not meet this behaviour, then $T$ is not quasianalytic, and so Hlat $T$ is non-trivial.

If the contraction $T$ is quasianalytic, then it is of class $C_{10}$, which means that $\lim _{n \rightarrow \infty}\left\|T^{* n} h\right\|=0<\lim _{n \rightarrow \infty}\left\|T^{n} h\right\|$ holds for all non-zero $h \in \mathcal{H}$; see Theorem 8 in $[\mathrm{KSz}$. Under this asymptotic behaviour, there is a connection between the spectrum $\sigma(T)$ of $T$ and the spectrum $\sigma(V)$ of $V$. First we note that $\sigma(V)$ is the essential support of $\omega(T): \sigma(V)=\operatorname{es}(\omega(T))$, which is the complement of the largest open set $\mathcal{O}$ on $\mathbb{T}$ with the property $m(\mathcal{O} \cap \omega(T))=0$. It can be easily proved that $\sigma(V)$ is neatly contained in $\sigma(T)$, that is $\sigma(V) \subset \sigma(T)$ and $m\left(\sigma(V) \cap \sigma^{\prime}\right)>0$ holds for every non-empty closed subset $\sigma^{\prime}$ of $\sigma(T)$ with the property that $\sigma(T) \backslash \sigma^{\prime}$ is also closed. More importantly, this is the only constraint on the spectrum of a $C_{10}$-contraction, even in the cyclic case, that is when $\vee_{n=0}^{\infty} T^{n} h=\mathcal{H}$ holds with some vector $h \in \mathcal{H}$; see Chapter IX in NFBK. Are there any other constraints if $T$ is quasianalytic? More precisely, we pose the following problem.

Question 1. Given a measurable set $\omega_{0} \subset \mathbb{T}$ of positive measure and a compact subset $\sigma$ of the closed unit disc $\mathbb{D}^{-}$such that es $\left(\omega_{0}\right)$ is neatly contained in $\sigma$, does a quasianalytic contraction $T$ exist with the properties $\sigma(T)=\sigma$ and $\omega(T)=\omega_{0}$ ?

In the $C_{10}$ class the construction starts by producing a $C_{10}$-contraction $T$ satisfying the conditions $\omega(T)=\omega_{0}$ and $\sigma(T)=\mathrm{es}\left(\omega_{0}\right)$, as a restriction of a bilateral weighted shift $W$ to an appropriately chosen invariant subspace. However, if $m\left(\mathbb{T} \backslash \omega_{0}\right)>0$ then $W$ is necessarily non-quasianalytic; otherwise $\pi(T)=\pi(W)=\mathbb{T}$ would happen. Furthermore, the coincidence $\sigma(T)=\operatorname{es}(\omega(T))$ is ensured by the condition $\sum_{n=1}^{\infty} n^{p}\left\|T^{-n}\right\|<\infty$ with some integer $p$. However, this relation implies the existence of an operator $C \in\{T\}^{\prime}$ and a non-zero continuous function $f$ on $\mathbb{T}$ such that $X C=f(V) X$ and the set $\{\zeta \in \omega(T): f(\zeta)=0\}$ has positive measure, 
less than $m(\omega(T))$; see Lemma IX.2.11 and its proof in [NFBK. Hence we can present a non-zero vector $h \in \mathcal{H}$ such that $X h$ is not cyclic for the commutant $\{V\}^{\prime}$, which is impossible if $T$ is quasianalytic; see Theorem 16 in Ker01. Therefore, we have to find another approach to provide a quasianalytic contraction $T$, if it exists at all, such that its spectrum $\sigma(T)$ is a proper subset of $\mathbb{T}$. First of all, the following simpler question should be answered.

Question 2. Do we have for every closed arc $J$ of positive measure on $\mathbb{T}$ and for every $c>0$ a quasianalytic contraction $T$ satisfying the conditions $\sigma(T)=\pi(T)=J$ and $\left\|T^{-1}\right\|>c$ ?

We know that the a.c. contraction $T$ has shift-type invariant subspaces if $\omega(T)=$ $\mathbb{T}$. Namely, $\mathcal{H}=\vee \operatorname{Lat}_{\mathrm{s}} T$, where $\operatorname{Lat}_{\mathrm{s}} T$ consists of those invariant subspaces $\mathcal{M}$ where $T \mid \mathcal{M}$ is similar to the simple unilateral shift $S \in \mathcal{L}\left(H^{2}\right), S f=\chi f(\chi(\zeta)=\zeta)$; see Theorem IX.3.6 in [NFBK]. Any quasianalytic contraction can be related to such a contraction having a rich invariant subspace lattice.

Theorem 2. For every quasianalytic contraction $T_{1}$, there exists a quasianalytic contraction $T_{2}$ with $\pi\left(T_{2}\right)=\mathbb{T}$ such that $\left\{T_{2}\right\}^{\prime} \supset\left\{T_{1}\right\}^{\prime}$ and so Hlat $T_{2} \subset$ Hlat $T_{1}$.

Proof. By Theorem 3 of KT12] there exist a compact set $K \subset \pi\left(T_{1}\right)$ and a continuous function $f$ on $\mathbb{D}^{-}$such that $f$ is analytic (even univalent) on $\mathbb{D}, f^{-1}(\mathbb{T})=K$ and $m(f(\alpha))=0$ for every Borel subset $\alpha$ of $K$ of zero measure. Then $\pi\left(T_{2}\right)=\mathbb{T}$ holds for the a.c. contraction $T_{2}=f\left(T_{1}\right)$ by Corollary 2.5 of [Ker11] (see also Lemma 5 in $[\mathrm{KT} 12]$ ). It is obvious that $\left\{T_{2}\right\}^{\prime} \supset\left\{T_{1}\right\}^{\prime}$.

Therefore, the (HSP) for a.n.v. contractions can be reduced to the case, when $T$ is quasianalytic and $\pi(T)=\mathbb{T}$. Clearly, $\mathbb{T}$ is neatly contained in $\sigma(T)$ exactly when $\sigma(T)$ is connected. Thus, in this particular class, Question 1 has the following modified form.

Question 3. Given a connected, compact subset $\sigma$ of $\mathbb{D}^{-}$, containing $\mathbb{T}$, does there exist a quasianalytic contraction $T$ satisfying the conditions $\sigma(T)=\sigma$ and $\pi(T)=\mathbb{T} ?$

The preceding two questions are related. Let $\mathbb{D}_{+}:=\{z \in \mathbb{D}: \operatorname{Im} z>0\}$, $\mathbb{T}_{+}:=\{\zeta \in \mathbb{T}: \operatorname{Im} \zeta \geq 0\}$, and for any $K \subset \mathbb{C}$ let $K^{2}:=\left\{z^{2}: z \in K\right\}$.

Theorem 3. A positive answer for Question 2 implies an affirmative answer for Question 3 in the special case, when $\sigma=K^{2}$ with a connected, compact set $K$ such that $\mathbb{T}_{+} \subset K \subset \mathbb{D}_{+}^{-}$.

Proof. Let $K$ be a connected, compact set such that $\mathbb{T}_{+} \subset K \subset \mathbb{D}_{+}^{-}$and $K^{2}=\sigma$. We apply the technique used in Section IX.2 of [NFBK] to obtain a quasianalytic contraction $\widetilde{T}$ satisfying the conditions $\sigma(\widetilde{T})=K$ and $\pi(\widetilde{T})=\mathbb{T}_{+}$.

Let $\left\{\lambda_{n}\right\}_{n=1}^{\infty}$ be a dense sequence in $K$. For every $n \in \mathbb{N}$, let us consider the connected, open set $\Omega_{n}=\{z \in \mathbb{C}: \operatorname{dist}(z, K)<1 / n\}$, and select a point $\lambda_{n}^{\prime} \in$ $\mathbb{D} \cap \Omega_{n}$ so that $\left|\lambda_{n}-\lambda_{n}^{\prime}\right|<1 /(2 n)$. Let $\Gamma_{n} \subset\left(\Omega_{n} \cap \mathbb{D}\right) \cup\{-1,1\}$ be a simple rectifiable curve, with endpoints -1 and 1 , such that the simply connected domain $G_{n}$ bounded by $\mathbb{T}_{+} \cup \Gamma_{n}$ is contained in $\Omega_{n}$ and $\lambda_{n}^{\prime} \in G_{n}$. There exists a conformal mapping $f_{n}: \mathbb{D} \rightarrow G_{n}$, having continuous extension onto $\mathbb{D}^{-}$, such that $f_{n}(0)=$ $\lambda_{n}^{\prime}$. Let us consider the closed arc $J_{n}=f_{n}^{-1}\left(\mathbb{T}_{+}\right)$. By our assumption there exists a quasianalytic contraction $T_{n} \in \mathcal{L}\left(\mathcal{H}_{n}\right)$ such that $\sigma\left(T_{n}\right)=\pi\left(T_{n}\right)=J_{n}$ 
and $\left\|T_{n}^{-1}\right\|>n$. Then $\widetilde{T}_{n}=f_{n}\left(T_{n}\right)$ is also a quasianalytic contraction with the properties $\sigma\left(\widetilde{T}_{n}\right)=\pi\left(\widetilde{T}_{n}\right)=\mathbb{T}_{+}$; see Proposition IX.2.4 in [NFBK] and Corollary 2.5 in Ker11.

Setting $\widetilde{T}=\sum_{n=1}^{\infty} \oplus \widetilde{T}_{n}$, we may verify that $\sigma(\widetilde{T})=K$ and $\pi(\widetilde{T})=\mathbb{T}_{+}$. Indeed, for every $n \in \mathbb{N}$, there exists a unit vector $e_{n} \in \mathcal{H}_{n}$ such that $\left\|T_{n} e_{n}\right\|<1 / n$. Since $f_{n}(z)-\lambda_{n}^{\prime}=z g_{n}(z)$, where $\left\|g_{n}\right\|_{\infty} \leq 2$, it follows that $\left\|\widetilde{T}_{n} e_{n}-\lambda_{n}^{\prime} e_{n}\right\|=$ $\left\|g_{n}\left(T_{n}\right) T_{n} e_{n}\right\| \leq 2 / n$. Taking into account that each $\lambda \in K$ is a cluster point of the sequence $\left\{\lambda_{n}^{\prime}\right\}_{n=1}^{\infty}$, we infer that $K \subset \sigma(\widetilde{T})$. On the other hand, if $\lambda \notin K$ then $\delta_{0}=\operatorname{dist}\left(\lambda, G_{n_{0}}\right)>0$ for some $n_{0} \in \mathbb{N}$. Thus, $\left\|\left(\widetilde{T}_{n}-\lambda I\right)^{-1}\right\| \leq\left\|1 /\left(f_{n}-\lambda\right)\right\|_{\infty} \leq$ $1 / \delta_{0}$ holds whenever $n \geq n_{0}$. Since $\lambda \notin \sigma\left(\widetilde{T}_{n}\right)$ for all $n$, it follows that $\lambda \notin \sigma(\widetilde{T})$. Therefore, $\sigma(\widetilde{T})=K$. Finally, $\pi(\widetilde{T})=\cap_{n=1}^{\infty} \pi\left(\widetilde{T}_{n}\right)=\mathbb{T}_{+}$is obvious. Now, $T=\widetilde{T}^{2}$ is a quasianalytic contraction satisfying the conditions $\sigma(T)=K^{2}=\sigma$ and $\pi(T)=$ $\mathbb{T}_{+}^{2}=\mathbb{T}$.

Remark 4. Not every connected, compact set $\mathbb{T} \subset \sigma \subset \mathbb{D}^{-}$can be represented as $\sigma=K^{2}$ with a connected, compact set $\mathbb{T}_{+} \subset K \subset \mathbb{D}_{+}^{-}$. Indeed, let $\rho:[0,1) \rightarrow[0, \infty)$ and $\varphi:[0,1) \rightarrow[0, \infty)$ be strictly increasing, continuous functions satisfying the conditions $\rho(0)=\varphi(0)=0, \lim _{t \rightarrow 1-} \rho(t)=1, \lim _{t \rightarrow 1-} \varphi(t)=\infty$, and take the connected compact set $\sigma=\mathbb{T} \cup\left\{\rho(t) e^{i \varphi(t)}: t \in[0,1)\right\}$.

The a.n.v. contraction $T$ is called asymptotically cyclic if its unitary asymptote $V$ is cyclic. In that case the commutant $\{T\}^{\prime}$ of $T$ can be identified with a subalgebra $\mathcal{F}(T)$ of $L^{\infty}(\mathbb{T})$, called the functional commutant of $T$. See [Ker11] and [KSz] for the study of $\mathcal{F}(T)$. Clearly, the (ISP) can be reduced to the case when $T$ is asymptotically cyclic. Therefore, it is important to know the spectral behaviour in this setting, too. We recall that $\mathcal{L}_{0}(\mathcal{H})$ stands for the set of asymptotically cyclic, quasianalytic contractions on $\mathcal{H}$, while $\mathcal{L}_{1}(\mathcal{H})=\left\{T \in \mathcal{L}_{0}(\mathcal{H}): \pi(T)=\mathbb{T}\right\}$. The same commutants arise in these two classes. Namely, the following statement was proved in KT12.

Proposition 5. For every $T_{0} \in \mathcal{L}_{0}(\mathcal{H})$, there exists $T_{1} \in \mathcal{L}_{1}(\mathcal{H})$ such that $\left\{T_{0}\right\}^{\prime}=$ $\left\{T_{1}\right\}^{\prime}$ and so Hlat $T_{0}=$ Hlat $T_{1}$.

This proposition makes it especially important to answer the following question.

Question 4. What are the possible spectra of the contractions belonging to $\mathcal{L}_{1}(\mathcal{H})$ ?

We know that for every $0 \leq \delta<1$ there is a contraction $T_{\delta} \in \mathcal{L}_{1}(\mathcal{H})$ such that $\sigma\left(T_{\delta}\right)=\{z \in \mathbb{C}: \delta \leq|z| \leq 1\}$; see Example 5.8 in Ker11 and Example 24 in $\mathrm{KSz}$. Now we show that the spectrum can be the unit circle $\mathbb{T}$ too. The following theorem also gives a positive answer for Question 2 in the special case, when the arc $J$ is the whole circle $\mathbb{T}$.

Theorem 6. For every $c>1$, there is a contraction $T \in \mathcal{L}_{1}(\mathcal{H})$ such that $\sigma(T)=\mathbb{T}$ and $\left\|T^{-1}\right\| \geq c$.

Proof. We present a bilateral weighted shift with the prescribed properties. (For their study see [Shi74] and Section 7 in $[\mathrm{KSz}$.$) Let \beta: \mathbb{Z} \rightarrow[1, \infty)$ be a sequence such that $\beta(n)=1$ for all $n \geq 0$ and $\beta(-n)=e^{\varphi(n)}$ for $n \in \mathbb{N}$, where the increasing sequence $\varphi: \mathbb{N} \rightarrow[1, \infty)$ with $\lim _{n \rightarrow \infty} \varphi(n)=\infty$ is specified later. Let $\sum_{n=-\infty}^{\infty} \widehat{f}(n) \chi^{n}$ stand for the Fourier series of the function $f \in L^{2}(\mathbb{T})$, where 
$\chi(\zeta)=\zeta(\zeta \in \mathbb{T})$. We consider the Hilbert space

$$
L^{2}(\beta)=\left\{f \in L^{2}(\mathbb{T}):\|f\|_{\beta}^{2}:=\sum_{n=-\infty}^{\infty}|\widehat{f}(n)|^{2} \beta(n)^{2}<\infty\right\}
$$

and the asymptotically cyclic $C_{10}$-contraction $T_{\beta} \in \mathcal{L}\left(L^{2}(\beta)\right)$, defined by $T_{\beta} f=$ $\chi f$.

Now we specify the sequence $\varphi$ so that $T_{\beta}$ is quasianalytic with $\sigma\left(T_{\beta}\right)=\mathbb{T}$. Select a strictly decreasing sequence $\left\{q_{k}\right\}_{k=1}^{\infty}$ of real numbers in $(0,1)$ such that $\lim _{k \rightarrow \infty} q_{k}=0$, and then select a strictly increasing sequence $\left\{p_{k}\right\}_{k=1}^{\infty}$ of positive integers satisfying the conditions $p_{1}=1$ and $\sum_{n=p_{k}+1}^{p_{k+1}} \frac{1}{n} \geq 1 / q_{k}$ for every $k \in \mathbb{N}$. Setting $c>1$, let $\varphi(1):=c$, and for any $k \in \mathbb{N}$ and $p_{k}<n \leq p_{k+1}$ let $\varphi(n):=$ $\varphi\left(p_{k}\right)+\left(n-p_{k}\right) q_{k}$. Clearly, $\varphi$ is increasing. It can be verified by induction that $\varphi\left(p_{k}\right) \geq p_{k} q_{k}$ holds for every $k \in \mathbb{N}$. Thus $\varphi(n) \geq n q_{k}$ if $p_{k}<n \leq p_{k+1}$, whence

$$
\begin{aligned}
\sum_{n=1}^{\infty} \frac{\log \beta(-n)}{n^{2}} & =\sum_{n=1}^{\infty} \frac{\varphi(n)}{n^{2}}=c+\sum_{k=1}^{\infty} \sum_{n=p_{k}+1}^{p_{k+1}} \frac{\varphi(n)}{n^{2}} \\
& \geq \sum_{k=1}^{\infty} q_{k} \sum_{n=p_{k}+1}^{p_{k+1}} \frac{1}{n} \geq \sum_{k=1}^{\infty} 1=\infty
\end{aligned}
$$

follows. We conclude that $T_{\beta}$ is quasianalytic, and so $T_{\beta} \in \mathcal{L}_{1}\left(L^{2}(\beta)\right)$; see Proposition 31 in $\underline{\mathrm{KSz}}$.

Since the sequence $\left\{q_{k}\right\}_{k=1}^{\infty}$ is decreasing, it follows that

$$
\left\|T_{\beta}^{-n}\right\|=\sup \left\{\frac{\beta(j-n)}{\beta(j)}: j \in \mathbb{Z}\right\}=e^{\varphi(n)} \quad \text { for all } n \in \mathbb{N} .
$$

In particular, we get $\left\|T_{\beta}^{-1}\right\|=e^{\varphi(1)}=e^{c} \geq c$. Furthermore, for every $k \in \mathbb{N}$ and $p_{k}<n \leq p_{k+1}$ we have

$$
0 \leq \frac{\varphi(n)}{n}=\frac{\varphi\left(p_{k}\right)}{n}+\frac{n-p_{k}}{n} q_{k} \leq \frac{\varphi\left(p_{k}\right)}{p_{k}}+q_{k} .
$$

Hence $\lim _{n \rightarrow \infty} \varphi(n) / n=0$ holds, if $\lim _{k \rightarrow \infty} \varphi\left(p_{k}\right) / p_{k}=0$. The inequality

$$
\frac{1}{q_{k}} \leq \sum_{n=p_{k}+1}^{p_{k+1}} \frac{1}{n} \leq \int_{p_{k}}^{p_{k+1}} \frac{\mathrm{d} x}{x}=\ln \frac{p_{k+1}}{p_{k}}
$$

yields that

$$
\frac{p_{k}}{p_{k+1}} \leq e^{-\frac{1}{q_{k}}} \leq \frac{1}{2}
$$

Applying the recursive formula

$$
\frac{\varphi\left(p_{k+1}\right)}{p_{k+1}}=\frac{\varphi\left(p_{k}\right)}{p_{k}} \cdot \frac{p_{k}}{p_{k+1}}+\left(1-\frac{p_{k}}{p_{k+1}}\right) q_{k}
$$

we can check by induction that $\varphi\left(p_{k}\right) / p_{k} \leq 2 c$ holds for all $k \in \mathbb{N}$. The previous inequalities imply that

$$
\frac{\varphi\left(p_{k+1}\right)}{p_{k+1}} \leq 2 c e^{-\frac{1}{q_{k}}}+q_{k}
$$


whence $\lim _{k \rightarrow \infty} \varphi\left(p_{k}\right) / p_{k}=0$ immediately follows. Therefore, the spectral radius of $T_{\beta}^{-1}$ is

$$
r\left(T_{\beta}^{-1}\right)=\lim _{n \rightarrow \infty}\left\|T_{\beta}^{-n}\right\|^{\frac{1}{n}}=\lim _{n \rightarrow \infty} e^{\varphi(n) / n}=1 .
$$

In view of the circular symmetry of $\sigma\left(T_{\beta}\right)$ we obtain that $\sigma\left(T_{\beta}\right)=\mathbb{T}$.

Relying on this statement we can provide contractions in $\mathcal{L}_{1}(\mathcal{H})$ with more sophisticated spectra.

Examples 7. (a) Given any $\delta \in(0,1)$, let us consider the domain $\Omega_{\delta}=\left\{z=r e^{i t}\right.$ : $\sqrt{\delta}<r<1$ and $0<t<\pi\}$. Let $\eta_{\delta}$ be a conformal mapping of $\mathbb{D}$ onto $\Omega_{\delta}$, and set $\vartheta_{\delta}=\eta_{\delta}^{2}$. If $T \in \mathcal{L}_{1}(\mathcal{H})$ is an operator with $\sigma(T)=\mathbb{T}$, then $T_{\delta}=\vartheta_{\delta}(T) \in \mathcal{L}_{1}(\mathcal{H})$ and $\sigma\left(T_{\delta}\right)=\mathbb{T} \cup \delta \mathbb{T} \cup[\delta, 1]$. Observe that $\mathbb{D} \backslash \sigma(T)$ is not connected.

(b) We recall that a domain $\Omega \subset \mathbb{C}$ is called a circular comb domain, if it is of the form $\Omega=\mathbb{D} \backslash\{r \zeta: \zeta \in H, \rho(\zeta)<r<1\}$, where $H \subset \mathbb{T}$ is countable and $\rho: H \rightarrow(0,1)$. Let $K$ be a Cantor-type compact set on $\mathbb{T}$ of positive measure. In view of Theorem 3 of KT12 we know that there exists a compact set $\widetilde{K} \subset K$ and a conformal mapping $f$ of $\mathbb{D}$ onto a circular comb domain $\Omega$ such that $f$ can be continuously extended onto $\mathbb{D}^{-}, f^{-1}(\mathbb{T})=\widetilde{K}$, and $m(f(\alpha))=0$ whenever $\alpha \subset \widetilde{K}$ is of measure zero. If $T \in \mathcal{L}_{1}(\mathcal{H})$ with $\sigma(T)=\mathbb{T}$, then the spectrum of $\widetilde{T}=f(T) \in \mathcal{L}_{1}(\mathcal{H})$ is $\sigma(\widetilde{T})=\mathbb{T} \cup\{r \zeta: \zeta \in H, \rho(\zeta) \leq r<1\}$, where $H$ is dense in $\mathbb{T}$. Questions $1-4$, in their full generality, remain open.

\section{REFERENCES}

[Ker01] László Kérchy, On the hyperinvariant subspace problem for asymptotically nonvanishing contractions, Recent advances in operator theory and related topics (Szeged, 1999), Oper. Theory Adv. Appl., vol. 127, Birkhäuser, Basel, 2001, pp. 399-422. MR1902813 (2003e:47013)

[Ker11] László Kérchy, Quasianalytic contractions and function algebras, Indiana Univ. Math. J. 60 (2011), no. 1, 21-40, DOI 10.1512/iumj.2011.60.4280. MR.2952408

[Ker13] László Kérchy, Unitary asymptotes and quasianalycity, Acta Sci. Math. (Szeged) 79 (2013), no. 1-2, 253-271. MR3100438

[KSz] László Kérchy and Attila Szalai, Asymptotically cyclic quasianalytic contractions, Studia Math. 223 (2014), no. 1, 53-76, DOI 10.4064/sm223-1-4. MR.3268717

[KT12] László Kérchy and Vilmos Totik, Compression of quasianalytic spectral sets of cyclic contractions, J. Funct. Anal. 263 (2012), no. 9, 2754-2769, DOI 10.1016/j.jfa.2012.08.016. MR.2967305

[NFBK] B. Sz.-Nagy, C. Foias, H. Bercovici, and L. Kérchy, Harmonic analysis of operators on Hilbert space, Revised and Enlarged Edition, Universitext, Springer, New York, 2010.

[Shi74] Allen L. Shields, Weighted shift operators and analytic function theory, Topics in operator theory, Amer. Math. Soc., Providence, R.I., 1974, pp. 49-128. Math. Surveys, No. 13. MR0361899(50 \#14341)

Bolyai Institute, University of Szeged, Aradi vértanúk tere 1, 6720 Szeged, Hungary

E-mail address: kerchy@math.u-szeged.hu

Bolyai Institute, University of Szeged, Aradi vértanúk tere 1, 6720 Szeged, Hungary

E-mail address: szalaiap@math.u-szeged.hu 